\title{
Binding partners regulate unfolding of myosin VI to activate the molecular motor
}

\author{
Ália dos Santos ${ }^{1 \#}$, Natalia Fili ${ }^{1 \#}$, Yukti Hari-Gupta ${ }^{2 \$}$, Rosemarie E. Gough ${ }^{1}$, Lin Wang ${ }^{3}$, \\ Marisa Martin-Fernandez, Jesse Aaron ${ }^{4}$, Eric Waite ${ }^{4}$, Teng-Leong $\mathrm{Chew}^{4}$ and \\ Christopher P. Toseland ${ }^{1 *}$ \\ ${ }^{1}$ Sheffield Cancer Centre, Department of Oncology and Metabolism, University of Sheffield, Sheffield, \\ S10 2RX, UK. ${ }^{2}$ School of Biosciences, University of Kent, Canterbury, CT2 7NJ, UK. ${ }^{3}$ Central Laser \\ Facility, Research Complex at Harwell, Science and Technology Facilities Council, Rutherford Appleton \\ Laboratory, Harwell, Didcot, Oxford, OX11 OQX, UK. ${ }^{4}$ Advanced Imaging Center, HHMI Janelia \\ Research Campus, Ashburn, USA. ${ }^{\$}$ Current address: MRC LMCB, University College London, Gower \\ Street, London, WC1E 6BT, UK. "Equal contribution.
}

*Corresponding Author: Christopher P. Toseland c.toseland@sheffield.ac.uk

Key Words: Myosin VI, Dab2, NDP52, molecular motor

\section{ABSTRACT}

Myosin VI is the only minus-end actin motor and is coupled to various cellular processes ranging from endocytosis to transcription. This multipotent nature is achieved through alternative isoform splicing and interactions with a network of binding partners. How the regulation mechanism of myosin VI varies between different isoforms and binding partners remains unexplored. Here, we have compared the regulation of two myosin VI splice isoforms by two different binding partners. By combining biochemical and single-molecule approaches, we propose that myosin $\mathrm{VI}$ regulation follows a generic mechanism, independently of the spliced isoform and the binding partner involved. We describe how myosin VI adopts an autoinhibited backfolded state which is unfolded by binding partners. This unfolding activates the motor and can subsequently trigger dimerization. We have further expanded our study by using live single molecule imaging to investigate the impact of binding partners upon myosin $\mathrm{VI}$ molecular organisation and dynamics. Overall, binding partners determine myosin VI function.

\section{INTRODUCTION}

Myosins are actin-based molecular motors which perform vital roles in numerous of cellular processes [1]. Myosin VI (MVI) is associated with several cellular functions, ranging from endocytosis to transcription [2-9]. MVI is unique, in that it is the only member of the myosin family with the ability to move towards the minus end of actin filaments [10]. The functional diversity of MVI relies on its association with various binding partners [11, 12].

MVI is comprised of a motor domain, followed by a neck region consisting of a unique insert, which confers the reverse directionality, and an IQ domain (Figure 1A). Both of these domains bind calmodulin. The $\mathrm{N}$-terminal tail domain contains three structural domains: a three-helix-bundle (amino acids 835-916)[13], a single-alpha-helix (amino acids 942-978), followed by a short coiled-coil [14]. The Cterminal tail domain consists of the globular cargo binding domain (CBD). In addition, two regions within the tail can be alternatively spliced resulting in a 31-residue insertion (large-insert, LI) proximal to the CBD, and/or an 8-residue insertion within the CBD (small-insert, $\mathrm{SI}$ ). This leads to four splice isoforms, the non-insert (NI), $\mathrm{SI}$, LI and LI+SI [15], each with distinct intracellular distributions and functions [15, 16]. For example, the $\mathrm{NI}$ isoform is able to enter the nucleus, whereas the $\mathrm{LI}$ is confined to the cell periphery [4].

The CBD domain enables interactions with several binding partners which control the intracellular localisation and function of MVI [1]. This diverse list of partners includes disabled-2 (Dab2) and nuclear dot protein 52 (NDP52). These partners specifically bind to one of the two established motifs within the CBD of MVI, the WWY and RRL, respectively [8, 17, 18]. Binding partner selectivity is driven by isoform splicing, whereby the LI encodes an alpha helix which occludes the RRL motif [19]. This prevents partners, such as NDP52, from interacting with the protein, and therefore interactions for this isoform can be driven by the WWY motif. In contrast, in the $\mathrm{NI}$ isoform, both motifs are available for binding. However, the RRL site displays higher affinity for partners over the WWY motif, in order to select for those interactions [6]. We have previously revealed that MVI non-insert can adopt a back-folded conformation, in which the CBD is brought into close proximity to the motor domain [4]. NDP52 then interacts through the RRL binding motif, which leads to unfolding and subsequent dimerization of MVI through an internal dimerization site. It is unknown if the structurally distinct LI isoform is regulated in the 
same manner, or whether the WWY site can trigger the same structural rearrangements in MVI.

To this end, we have now expanded our studies to assess whether this mechanism applies generally to myosin VI, independent of partner and isoform preference. We have also further explored the molecular basis for this mechanism, and investigated the cellular organisation and dynamics with respect to binding partners. Overall, we present a detailed generic model governing the activation of myosin VI from an inactive back-folded state to an active unfolded motor capable of dimerization.

\section{RESULTS}

\section{Myosin VI back-folding is independent of isoform}

We have previously shown that the NI-MVI isoform is back-folded in vitro and in cells [4]. To address whether back-folding is a generic feature of $\mathrm{MVI}$, independent of the isoform, we investigated the conformation of LI-MVI. We utilised a previously employed FRET-based assay $[4,6,20]$ by titrating Alexa555-CBD against FITC-MVI814-1091 (containing the LI) to assess whether there is an interaction between the $\mathrm{N}$ - and $\mathrm{C}$-terminal tail domains, as would occur in a back-folded state. A significant concentration-dependent change in FRET was measured, indicating that the two domains are in close proximity (Figure 1B). The same was observed for the $\mathrm{NI}$ isoform $\mathrm{MVI}_{814-1060}$ (Figure 1C), consistent with previous results [4]. The calculated equilibrium dissociation constants $\left(K_{d}\right)$ corresponding to these data were $5.98(+/-0.58)$ $\mu \mathrm{M}$ and $4.8(+/-0.61) \mu \mathrm{M}$ for LI- and NI-MVI, respectively. These are relatively low micromolar affinities, suggesting the interactions are likely to be dynamic.
A

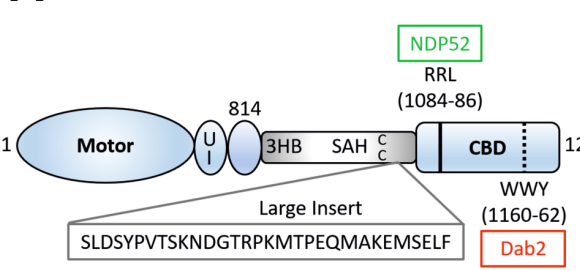

D



B

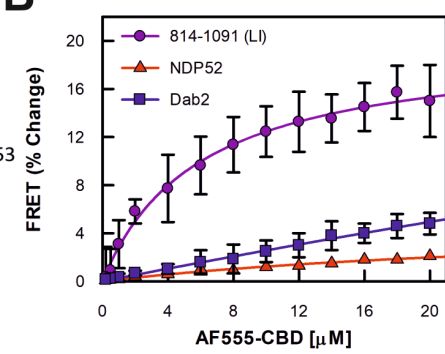

E

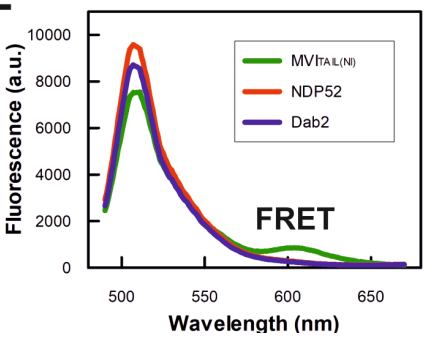

C

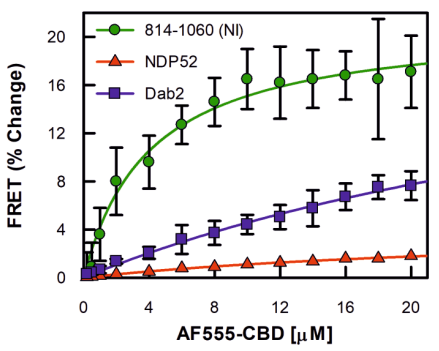

$\mathbf{F}$

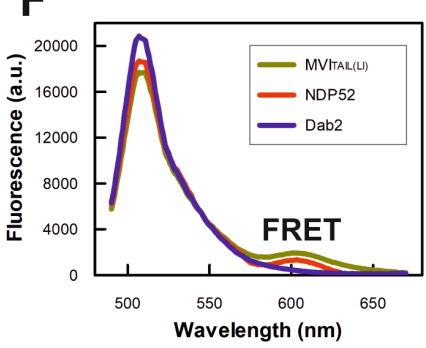

Figure 1: Backfolding of the myosin VI tail

(A) Cartoon depiction of the key regions of MVI, as discussed in the text. UI Unique Insert; 3HB Three Helix Bundle; SAH Stable Alpha Helix; CC Coiled-coil; CBD Cargo Binding Domain. This highlights position of the large insert (LI), along with NDP52 and Dab2 binding sites. (B) FRET titration of Alexa555-CBD against $1 \mu \mathrm{M}$ of FITC-

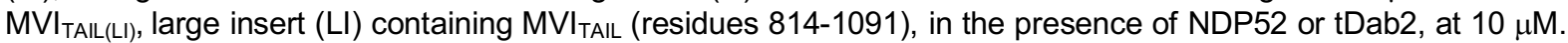
(C) FRET titration of Alexa555-CBD against $1 \mu \mathrm{M}$ of FITC-MVI TAIL(NI) non-insert (NI) MVI TAIL (residues 814-1060), in the presence of NDP52 or tDab2, at $10 \mu \mathrm{M}$. All titration data fitting was performed as described in Methods (Error bars represent SEM from three independent experiments). (D) Schematic representation of FRET assay to measure backfolding of the MVI TAlL. (E) Representative fluorescence spectra of $1 \mu \mathrm{M}$ GFP-MVI $\mathrm{IAIL}_{\text {(N) }}-\mathrm{RFP}+/-5$

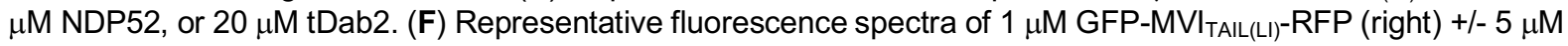
NDP52, or tDab2. 


\section{Binding partners associate to back-folded myosin $\mathrm{VI}$ and trigger unfolding.}

We have previously shown [4] that unfolding of the NI tail is directly driven by NDP52 following its interaction with the RRL motif and not through Calcium-Calmodulin interactions, as proposed by a different study [21], and we have previously addressed this discrepancy [4]. To address whether regulation of backfolding by binding partners is a generic mechanism, we followed a similar approach for both $\mathrm{LI}$ and $\mathrm{NI}$ isoforms, but now focusing upon the WWY partner of MVI, Dab2. Due to the instability of recombinant fulllength Dab2, we used a recombinant C-terminal truncation of the protein (residues 649-770), which contains the MVI binding site [8], as performed previously [6]. This truncation of Dab2 will be referred to as tDab2 throughout the manuscript. To assess whether Dab2 can regulate back-folding, the FRET assay between the CBD and the $\mathrm{MVI}_{814-1091}$ (LI) or the $\mathrm{MVI}_{814-1060}$ $(\mathrm{NI})$ tail was repeated following pre-incubation of the CBD with an excess of tDab2. As with NDP52, tDab2 sequestered the CBD, preventing the interaction between the two domains (Figures $1 \mathrm{~B}$ and $1 \mathrm{C}$ ), suggesting that Dab2 is also able to disrupt the intramolecular back-folding in both isoforms. However, tDab2 was not as efficient as NDP52, which is consistent with its weaker affinity for MVI [6].

To further confirm the direct effect of binding partners upon MVI unfolding, we used the FRET-based MVI tail conformation reporter, which we have previously developed [4]. Our reporter was based on a GFP-RFP FRET pair with the MVITAIL(NI) or MVITAlL(LI), placed in the middle (Figure 1D). As shown by the fluorescent spectra, a high FRET population was observed with both of these reporters, supporting the conclusion that the tails of both isoforms have the ability to back-fold (Figure 1E and $1 \mathrm{~F}$ ). The addition of $5 \mu \mathrm{M}$ NDP52 to the MVI TAIL(NI) FRET reporter, resulted in loss of the high FRET population. Similarly, $5 \mu \mathrm{M}$ tDab2 was able to deplete the FRET population of the MVITAIL(LI). However, $20 \mu \mathrm{M}$ of tDab2 were required to induce an equivalent effect on $\mathrm{MVI}_{\text {TAIL(NI), given its low }}$ affinity for this tail [6]. Also as expected, NDP52 induced little, if any, loss of the high FRET population of the $\mathrm{MVI}_{\mathrm{TAIL}(\mathrm{LI})}$ reporter, given that the RRL site is masked by the LI [19].

Altogether, these data demonstrate that the intramolecular backfolding of $\mathrm{MVI}$ is not isoform specific, but rather an intrinsic feature of the protein. Moreover, binding partners interacting at either motif can regulate this backfolding by triggering the unfolding step.

Stopped-flow transient kinetics were then employed to further explore the role of binding partner interactions during the unfolding process.
In particular, we endeavoured to determine whether binding partners first interact with the backfolded $\mathrm{MVI}$ triggering its unfolding, or whether they bind to spontaneously unfolded MVI stabilising the conformation. Given that both MVI isoforms showed the same response for either binding partner tested, further experiments only focused upon the $\mathrm{NI}$ isoform and NDP52.

First, FITC-MVITAIL(NI) and AF555-NDP52 were used as a FRET pair to report upon the interaction. $1 \mu \mathrm{M}$ FITC-MVITAIL(NI) was mixed with excess AF555-NDP52 under pseudo-first order conditions (Figure 2A). The fluorescence traces were characterised by two phases: an increase in FRET signal, followed by a partial decrease (Figure $2 \mathrm{~B}$ ). The first phase was fitted to a signal exponential function (Figure $2 \mathrm{C}$ ) and the observed rate constant was found to be dependent on the concentration of NDP52 (Figure 2D). We extracted an association rate constant of $1.72 \mu \mathrm{M}^{-1} \mathrm{~s}^{-1}$ and dissociation rate constant of $3.3 \mathrm{~s}^{-1}$, giving a $K_{\mathrm{d}}$ of $1.9 \mu \mathrm{M}$. This is consistent with the Equilibrium Dissociation constant previously derived from titrations [6]. The second phase in all three traces was also fitted to a single exponential function (Figure 2C), however the derived rate constants (average 2.1 $\mathrm{s}^{-1}$ ) were independent of NDP52 concentration (Figure 2D). This indicates that the second phase corresponds to a first order process, such as a conformation change. We therefore propose that these biphasic traces directly report upon a twostep process: first, binding of the partner onto backfolded $\mathrm{MVI}$, corresponding to the initial increase in FRET, and second, the subsequent unfolding of the myosin, corresponding to the consequent decrease in FRET due to a greater distance between the donor-acceptor dyes (Figure 2E). This suggests that direct binding of the unfolded MVI is unlikely to occur.

To further understand this partnerinduced conformation change, we used fluorescently labelled calmodulin bound to the $\mathrm{MVI}_{\text {TAIL(NI) }}$ as an environmental reporter. This approach would enable the probe to report upon either the partner binding step or the MVI unfolding step, or both processes (Figure $2 \mathrm{~F}$ and G). Cy3B-calmodulin was pre-mixed with MVITAIL(NI) in a 2:1 molar excess. $1 \mu \mathrm{M}$ Cy3Bcalmodulin MVITAIL(NI) was then mixed against an excess of non-fluorescent NDP52, under pseudofirst order conditions. A single exponential decrease in fluorescence was observed for all concentrations tested (Figure 2F), suggesting a single step process. Interestingly, the derived rate constants $\left(2 \mathrm{~s}^{-1}\right)$ were independent of NDP52 concentration (Figure $2 \mathrm{H}$ ) and were identical to the rate of constants of the second exponential phase observed in the AF555-NDP52 experiments (Figure 2D). We therefore propose 
bioRxiv preprint doi: https://doi.org/10.1101/2020.05.10.079236; this version posted May 10, 2020. The copyright holder for this preprint (which was not certified by peer review) is the author/funder, who has granted bioRxiv a license to display the preprint in perpetuity. It is made available under aCC-BY-NC-ND 4.0 International license.

that the Cy3B-calmodulin probe reports upon unfolding. This is not unanticipated because unfolding would lead to the largest local environmental change for the dye, as the partner itself binds to the CBD not the neck region. These observations are consistent with the partners not binding to a spontaneously unfolded MVI but rather to its backfolded conformation, which then triggers the unfolding of the protein (Figure 2E).

\section{Dimerization is an intrinsic property of myosin VI}

Unfolding of the $\mathrm{NI}$ isoform subsequently exposes dimerization sites, leading to protein oligomerization [4], similar to the LI isoform with binding partners [6]. There is a debate as to whether MVI dimerizes intrinsically or through a binding partner mediator [4, 13, 22-24]. We have previously reported that a tail region ahead of the CBD (Figure $3 \mathrm{~A}$ ) of $\mathrm{MVI}$ can dimerize independently of binding partners, but we also suggested that this region is blocked until interactions with binding partners occur [4].

NDP52 is a dimeric protein (Figure 3B) and therefore capable of dimerizing MVI with one CBD bound to each monomer. However, tDab2, which also has been previously shown to be able to dimerize MVI [6], is monomeric (Figure 3B). This finding reinforces our previous conclusion that dimerization is an intrinsic property of MVI.
A

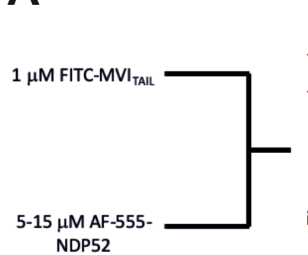

C

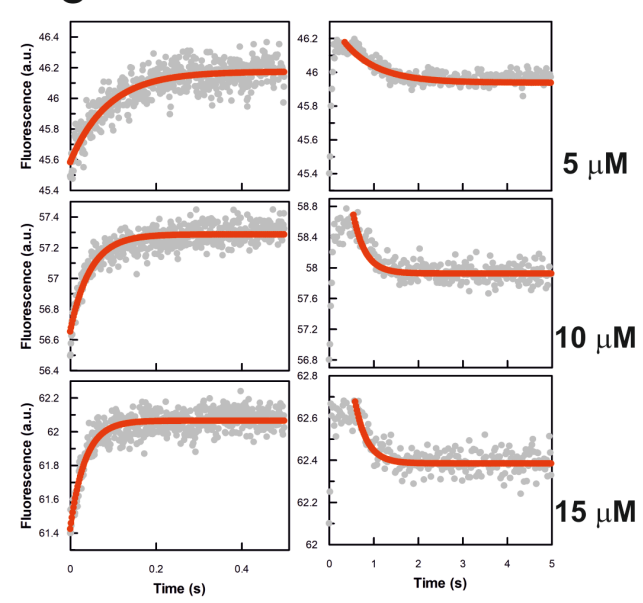

B

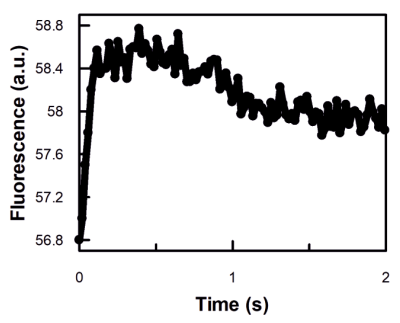

\footnotetext{
$\mathbf{F}$
}
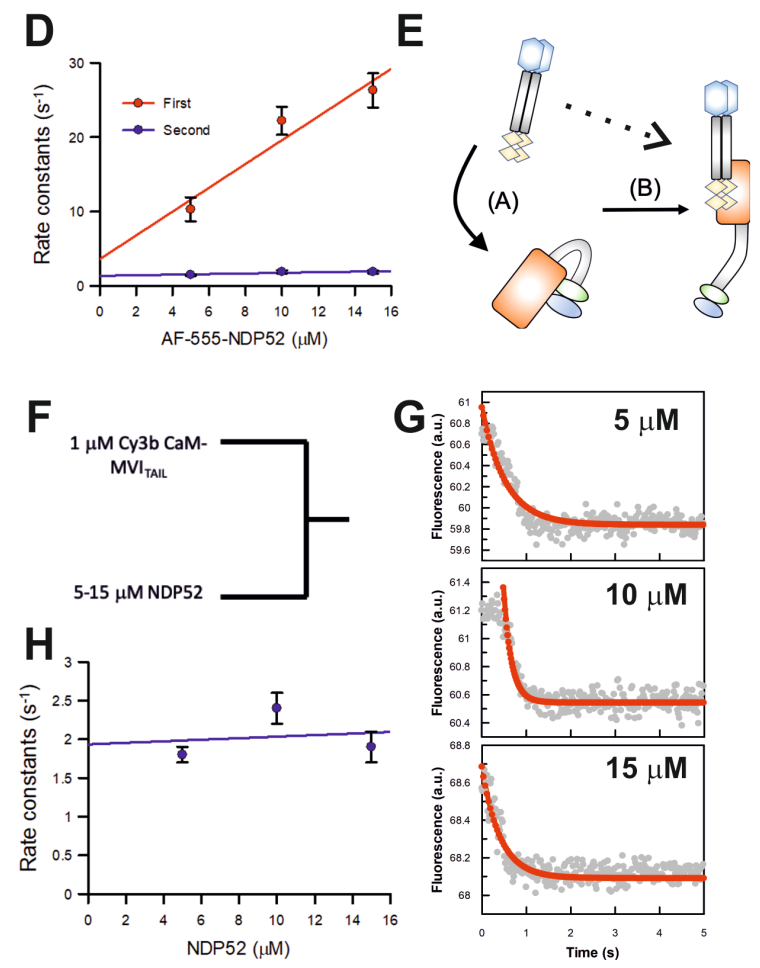

Figure 2: NDP52 association kinetics with the myosin VI tail.

(A) Schematic of the experiments for rapid mixing of the FITC labelled MVI TAIL and Alexa-Fluor555 labelled NDP52. Experiments were performed as described in the methods. (B) Representative stopped-flow fluorescence trace depicting both changes in FRET signal, as described in the text. (C) Representative stopped-flow fluorescence traces and exponential fitting to both transitions when the FITC-MVI TAIL is mixed with the stated concentrations of NDP52. (D) The individual traces were fitted to single exponentials and the dependence of the rate constants on concentration was then fitted to a straight line, as shown. The points shown are averages of at least 3 measurements, where error bars represent SEM. The fit for the first phase gives a slope of $1.72 \mu \mathrm{M}^{-1} \mathrm{~s}^{-1}$ and an intercept of $3.3 \mathrm{~s}^{-1}$. The second phase is independent of NDP52 concentration and gives an average rate constant of $2.1 \mathrm{~s}^{-1}$. (E) Cartoon depicting the two processes reported by the stopped-flow experiments. Step A represents NDP52 binding to the MVI TAIL, which is dependent upon NDP52 concentration. Step B represents the subsequent unfolding of MVI with a rate constant of $2.1 \mathrm{~s}^{-1}$, which is independent of NDP52 concentration. The dotted line represents an alternative model where NDP52 would bind to spontaneously unfolding MVI $\mathrm{TAIL}_{\text {. }}$ (F) Schematic of the experiments for rapid mixing of $1 \mu \mathrm{M}$ Cy3B-calmodulin-bound $\mathrm{MVI}_{\text {TAIL(NI) }}$ (pre-mix molar ratio 2:1) and unlabelled NDP52. Experiments were performed as described in the methods. (G) Representative stopped-flow fluorescence traces and exponential fitting to the fluorescence decrease when Cy3B-calmodulin $\mathrm{MVI}_{\mathrm{TAIL}(\mathrm{NI})}$ is mixed with the stated concentrations of NDP52. (H) The individual traces were fitted to single exponentials and the dependence of the rate constants on NDP52 concentration was then fitted to a straight line, as shown. The points shown are averages of at least 3 measurements, where error bars represent SEM. The rate constants are independent of NDP52 concentration, with an average value of $2 \mathrm{~s}^{-1}$. 
This allowed us to estimate that the complex would consist of one binding partner (monomer or dimer) per MVI molecule. For example, we would expect 4 NDP52 molecules (or 2 NDP52 dimers) in complex with $2 \mathrm{MVI}$ proteins.

To explore this hypothesis, we performed ATPase measurements on two MVI constructs, namely $\mathrm{MVI}_{1-814}$ and $\mathrm{MVI}_{1-1060}$, with the latter lacking the $\mathrm{CBD}$ and therefore having the proposed dimerization region exposed (Figure $3 C)$. The ATPase rates for $\mathrm{MVI}_{1-814}\left(k_{\text {cat }} 4.4 \mathrm{~s}^{-1}\right)$ were similar to full-length $\mathrm{MVI}\left(k_{\mathrm{cat}} 4.1 \mathrm{~s}^{-1}\right)$, while $\mathrm{MVI}_{1-1060}$ displayed a lower ATPase rate $\left(k_{\text {cat }} 2.79\right.$ $\mathrm{s}^{-1}$ ), as expected for a dimeric protein. This occurs due to molecular gating, whereby the ATPase activity of the individual motors is coordinated so that only a single motor turns over ATP at any given moment [25]. This rate was also similar to those measured for full-length $\mathrm{MVI}$ in the presence of dimeric NDP52 $\left(k_{\text {cat }} 2.11 \mathrm{~s}^{-1}\right)$ and monomeric tDab2 $\left(k_{\text {cat }} 2.55 \mathrm{~s}^{-1}\right)$. Overall, these ATPase measurements show that dimerization is a feature of MVI and not due to binding partner crosslinking. In addition, size-exclusion chromatography strongly supported the formation of $\mathrm{MVI}_{1-1060}$ dimers. Full-length MVI eluted as a single peak around $13 \mathrm{ml}$, whereas $\mathrm{MVI}_{1-814}$ eluted at $15.6 \mathrm{ml}$ (Figure 3D), consistent with this construct being smaller in size. However, $\mathrm{MVI}_{1}$. 1060 eluted earlier than both full length MVI and $\mathrm{MVI}_{1-814}$ constructs, suggesting it has adopted a distinct structure which supports its dimeric nature observed in the ATPase measurements.
Experiments were also performed with $\mathrm{MVI}$ and NDP52, however the proteins separated during the chromatography so a complex could not be resolved. Finally, size-exclusion chromatography also showed that $\mathrm{MVI}_{814-1060}$ elutes in two equal peaks, while $\mathrm{MVI}_{\mathrm{TAL}(\mathrm{NI})}$ and $\mathrm{CBD}$ elute as single species (Figure 3E), further supporting our finding that dimerization occurs within amino acids 814-1060. Taken together, the ATPase and size-exclusion chromatography data further support the presence of a dimerization site between amino acids 814-1060, consistent with our previous FRET experiments $[4,6]$.

Lastly, in order to follow the binding partner induced dimerization in real-time, we performed a FRET-based stopped flow assay. 1 $\mu \mathrm{M}$ FITC-MVITAIL(NI) and $1 \mu \mathrm{M}$ AF555-MVITAIL(NI) were pre-mixed, before being mixed in the stopped-flow with an excess of NDP52, under pseudo-first order conditions (Figure 4A). The fluorescence trace revealed a single exponential increase in FRET signal, with an observed rate constant of $2.2 \mathrm{~s}^{-1}$ (Figure 4B). This was independent of NDP52 concentration (Figure $5 \mathrm{C}$ ), but importantly, it was similar to the unfolding kinetics of the $\mathrm{MVI}_{\mathrm{TAl}(\mathrm{NI})}$ (Figure $2 \mathrm{C}$ and 2D). Altogether, we propose that dimerization is an intrinsic property of $\mathrm{MVI}$ and is a rapid process that occurs once unfolding has been triggered by the interaction with the binding partners, exposing the otherwise masked dimerization site (Figure 4D).

\section{A}

B
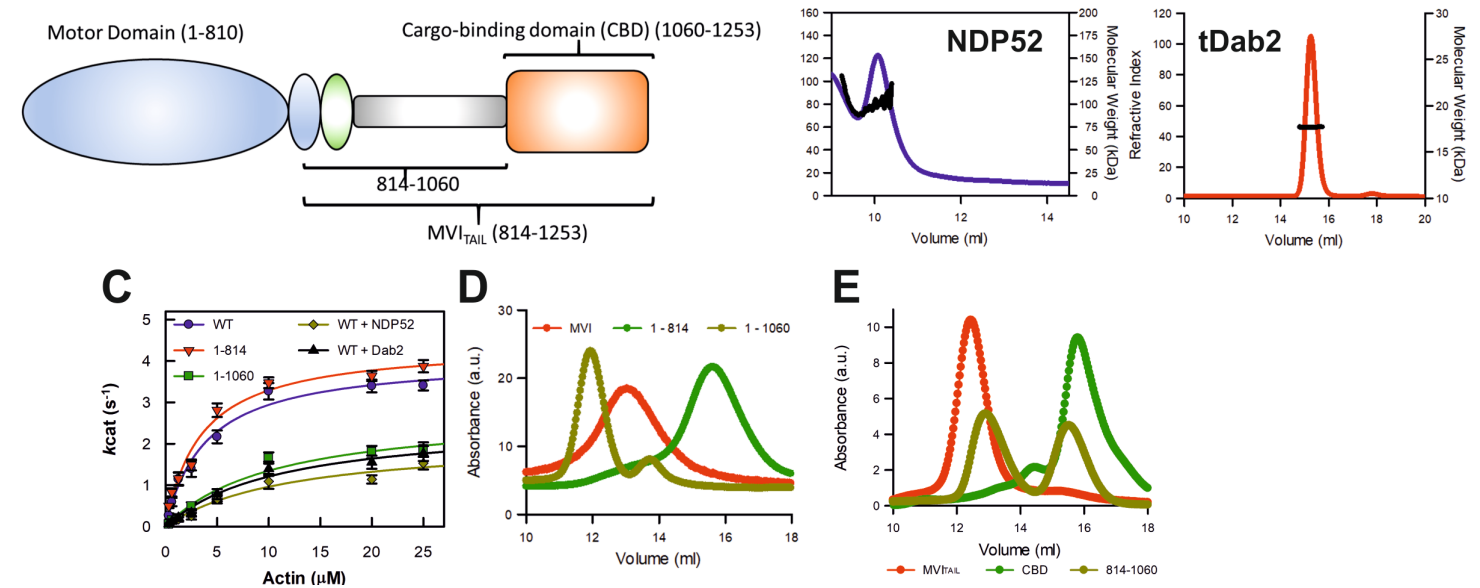

D

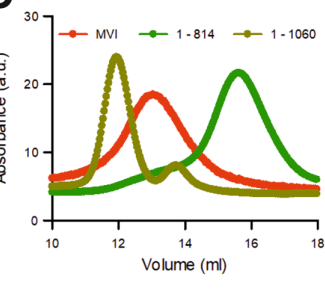

E

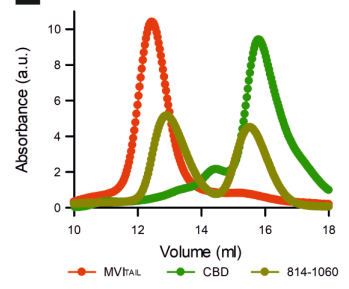

Figure 3: Dimerization of myosin VI.

(A) Cartoon depiction of the key domains of MVI which are used in the ATPase and size-exclusion chromatography measurements. (B) Representative SECMALS traces for NDP52 and tDab2 giving molecular weights of $102 \mathrm{kDa}$ and $17 \mathrm{kDa}$, respectively. This corresponds to dimeric NDP52 and monomeric tDab2. (C) Michaelis-Menten plot displaying steady-state actin-activated ATPase activity for the MVI constructs. Error bars represent SEM from three-independent experiments. (D) Representative SEC traces for $1 \mathrm{mg} / \mathrm{ml}$ of the MVI constructs. (E) Representative SEC traces for $1 \mathrm{mg} / \mathrm{ml}$ of the $\mathrm{MVI}_{\text {TAIL }}$ constructs. 
A

$1 \mu \mathrm{M}$ FITC-

$\mathrm{MVI}_{\text {TAII }} / \mathrm{AF}-555-$

$M \mathrm{MI}_{\text {TAIL }}$

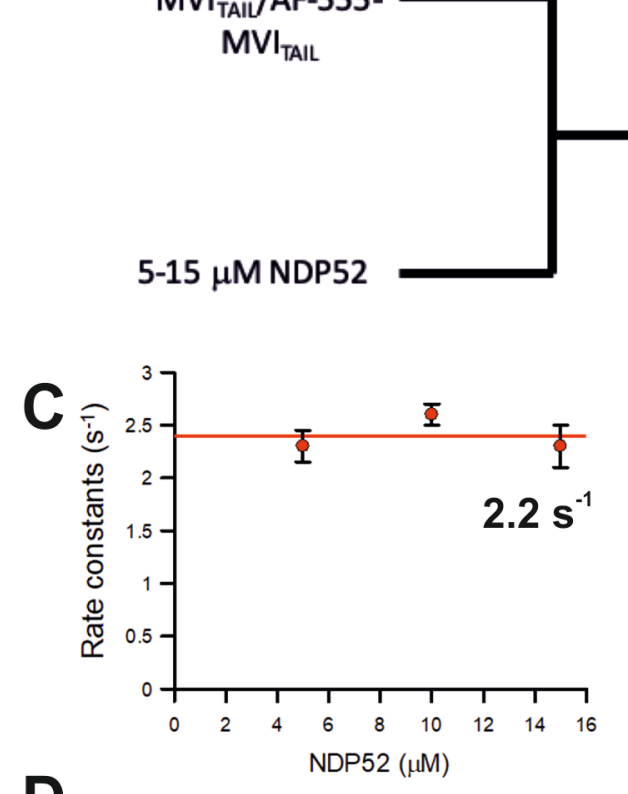

D

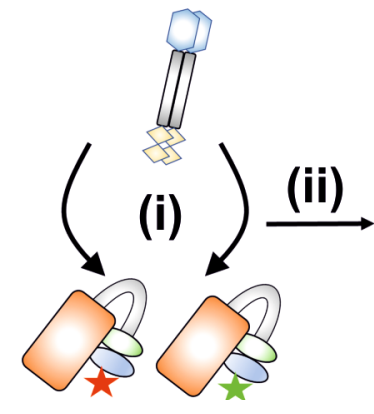

B
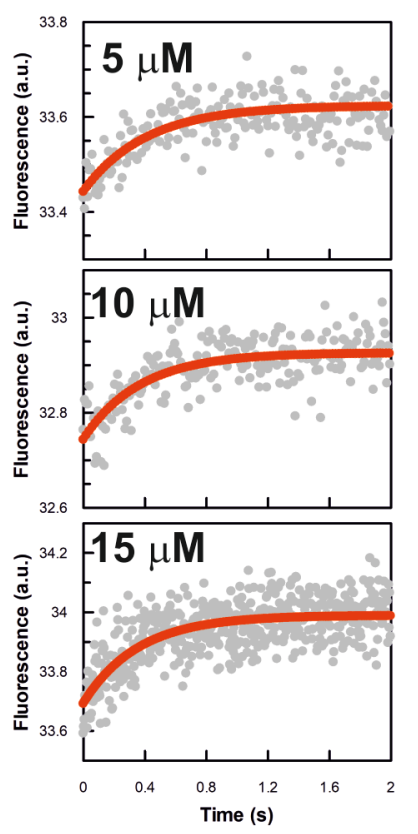

(iii)

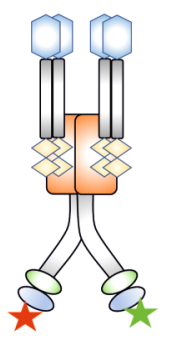

Figure 4: Binding partner driven dimerization of Myosin VI.

(A) Schematic of the experiments for rapid mixing of premixed FITC-MVI $\mathrm{IAIL}_{\text {and }}$ alexa-Fluor555--MVI $\mathrm{I}_{\text {TAlL }}$ with unlabelled NDP52. Experiments were performed as described in the methods. (B) Representative stopped-flow fluorescence traces and exponential fitting to the fluorescence increase, when the two labelled pools of MVI TAlL $_{\text {are }}$ mixed with the stated concentrations of NDP52. (C) The individual traces were fitted to single exponentials and the dependence of the rate constants on concentration was then fitted to a straight line, as shown. The points shown are averages of at least 3 measurements and error bars represent SEM. The rate constants are independent of NDP52 concentration, with an average value of $2.2 \mathrm{~s}^{-1}$. (D) Cartoon depicting binding partner driven dimerization based upon the experiments in Figure 2, 3 and 5. Both pools of labelled MVI TAL are folded. NDP52 binds to either tail (i) and then triggers their unfolding (ii). This then enables dimerization of the $\mathrm{MVI}_{\text {TAlL }}$ domains (iii).

\section{Clustering of myosin VI generates high local densities within the cell}

We have now defined the molecular mechanism underpinning $\mathrm{MVI}$ unfolding and dimerization. However, the affinities determined here and previously [6] are in the low micromolar range. Whilst it is possible that these interactions are part of large multi-valent complexes which enhance the overall complex affinities, the biochemical constants defined here suggest that binding partner interactions and subsequent dimerization would be rare events. We therefore focused upon investigating the in cellulo spatial organisation of $\mathrm{MVI}$ and NDP52 using super resolution imaging - Stochastic Optical Reconstruction Microscopy (STORM).
Widefield imaging against endogenous NDP52 and MVI showed that both proteins are distributed throughout HeLa cells (Figure 5A). STORM imaging of both proteins resolved NDP52 and MVI clusters (Figure 5B). To determine whether this distribution is indeed clustered or random (Figure $5 \mathrm{C}$ ), we performed cluster analysis using the linearized form of Ripley's K function [26] $L(r)-r$, where $r$ is the radius. A plot of $L(r)-r$ versus $r$ gives a value of zero for a random distribution (blue spots and line), but deviates from zero, due to molecular clustering (Figure 5C). This analysis showed that both NDP52 and MVI assemble into clusters, rather than being randomly distributed. To further understand this clustering behaviour, we used the Clus-DoC software [26], which allows to 
bioRxiv preprint doi: https://doi.org/10.1101/2020.05.10.079236; this version posted May 10, 2020. The copyright holder for this preprint (which was not certified by peer review) is the author/funder, who has granted bioRxiv a license to display the preprint in perpetuity. It is made available under aCC-BY-NC-ND 4.0 International license.

quantify the spatial distribution of a protein by generating cluster maps (Figure 5D). In this way, we calculated that, in the cytoplasm, $55(+/-16)$ $\%$ and $37.5(+/-14) \%$ of MVI and NDP52 molecules are in clusters (Figure 5E), respectively.

To explore the interaction between MVI and NDP52 within the cell, we performed colocalization analysis of the clusters (Figure 6). Colocalization can be represented by transforming the STORM images (Figure $6 \mathrm{~A}$ and $6 \mathrm{~B}$ ) into colocalization heat maps (Figure $6 \mathrm{C}$ and $6 \mathrm{D})$, which assign a different colour to the clusters of each protein depending on the level of colocalization between them. Exploring the cluster data further revealed that, for each protein, the colocalized clusters represent approximately $4 \%$ of all clusters (colocalised and non-colocalised) (Figure 6E). This is not a surprise given that both proteins interact with various other partners and are also involved in distinct pathways. However, the MVI colocalized


D

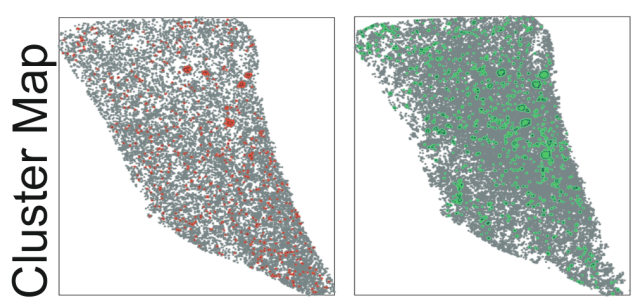

clusters are 10-fold larger than the noncolocalized ones, and contain three-times more molecules. For NDP52, the colocalized clusters are 8-fold larger than the non-colocalized ones, however they contain almost 2-fold less molecules. These results highlight differences in the clustering behaviour of the two proteins that might relate to the underlying cellular environment in which they interact.

Taken together, the cluster analysis suggests that molecular clustering of MVI does occur within the cell and is promoted by binding partners. As clustered MVI does not represent freely diffusing molecules, the high local density generated by clustering would enhance the impact of its biochemical properties. Within the confined and denser environment of the clusters, the low affinity interactions of MVI with its partners are more likely to occur. In this way, clustering would ensure that the subsequent unfolding and dimerization of $\mathrm{MVI}$ is readily facilitated and implemented, when needed.

\section{C}

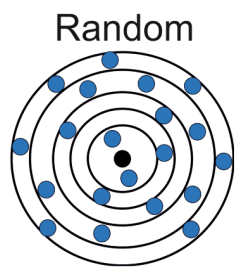

Clustered

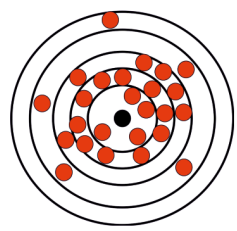

E

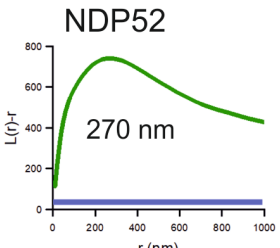

$\mathrm{MVI}$
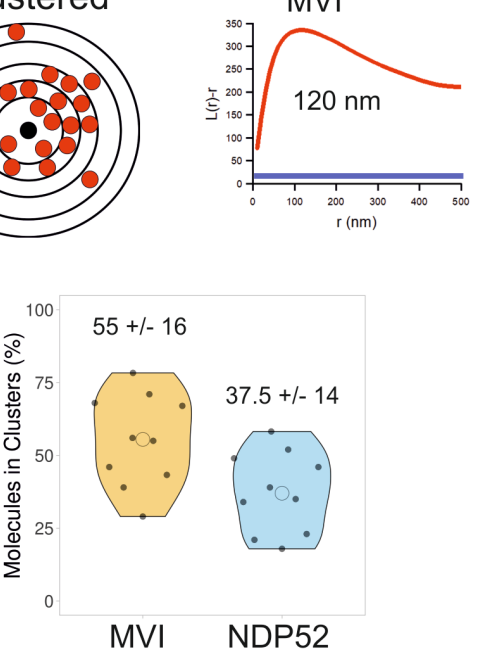

Figure 5: Cellular clustering of myosin VI and NDP52

(A) Widefield Immunofluorescence imaging against endogenous NDP52 (green) and MVI (red) in the cytoplasm of HeLa cells (Scale bar $10 \mu \mathrm{m}$ ). (B) STORM render images of the cells shown in (A) (Scale bar $10 \mu \mathrm{m}$ ). Images were acquired as described in the methods. (C) Depiction of a theoretical example of molecular clustering and random distribution. Molecular clustering is assessed by the linearized plot of Ripley's K function $L(r)-r$, versus $r$, where $r$ is the radius. Distribution can be plotted as an L-function where a randomly distributed set of molecules is a flat line. The green curve corresponds to the organisation of NDP52 (peak at $r=270 \mathrm{~nm}$ ) and the red curve corresponds to the organisation of MVI (peak at $r=120 \mathrm{~nm}$ ). (D) Cluster maps based upon the STORM render in (B). Clusters are shown in Red (NDP52) and Green (MVI). (E) Cluster analysis representing the percentage of molecules in a cluster. Individual data points correspond to the average number of molecules per cluster in the selected ROI, in an individual cell. The values represent the mean from all the ROIs for each protein $(n=10)$. 
bioRxiv preprint doi: https://doi.org/10.1101/2020.05.10.079236; this version posted May 10, 2020. The copyright holder for this preprint (which was not certified by peer review) is the author/funder, who has granted bioRxiv a license to display the preprint in perpetuity. It is made available under aCC-BY-NC-ND 4.0 International license.

A

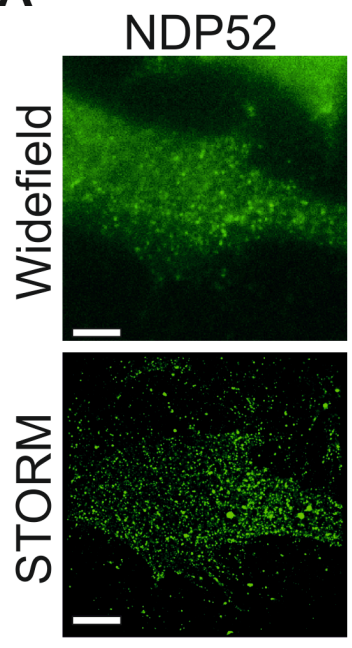

B
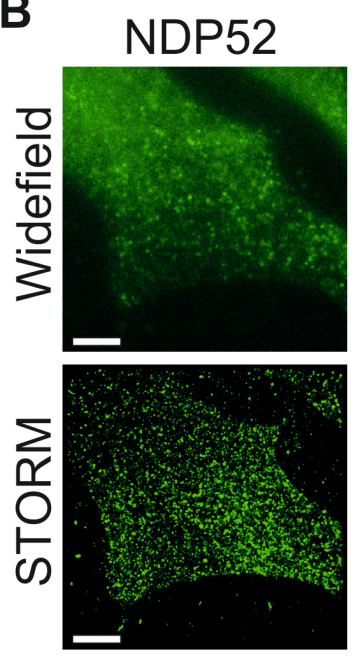

MVI
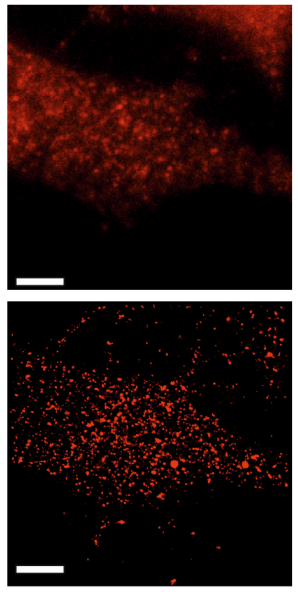

$\mathrm{MVI}$
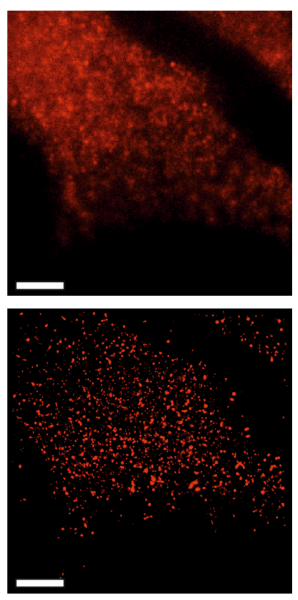
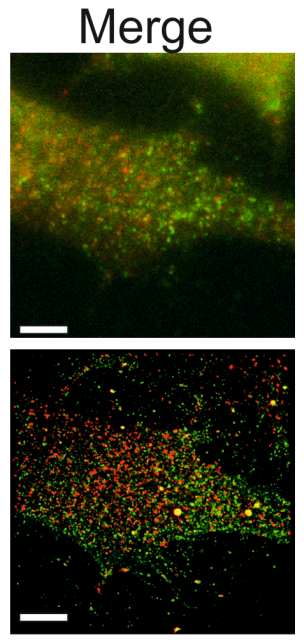

Merge
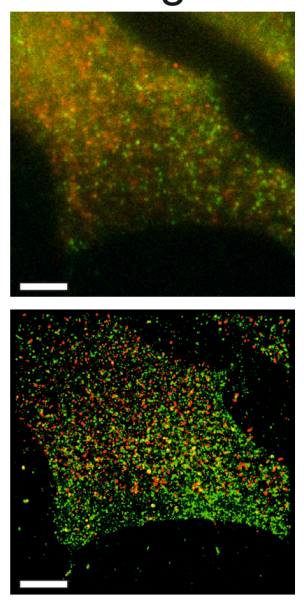

C
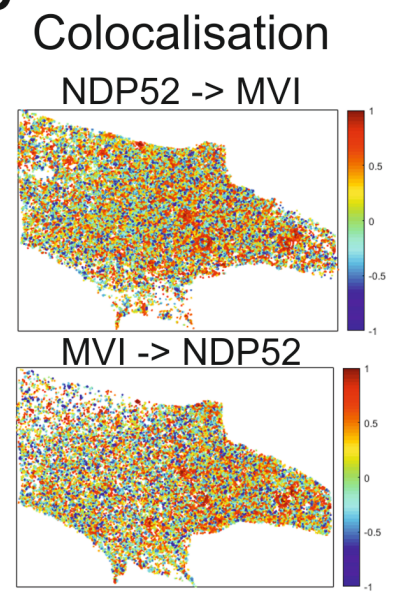

D Colocalisation



$\mathrm{MVI}$-> NDP52

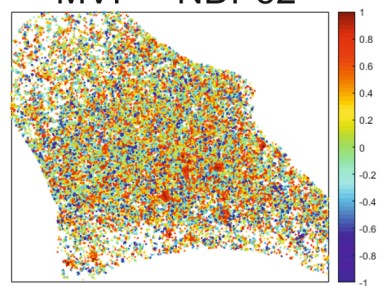

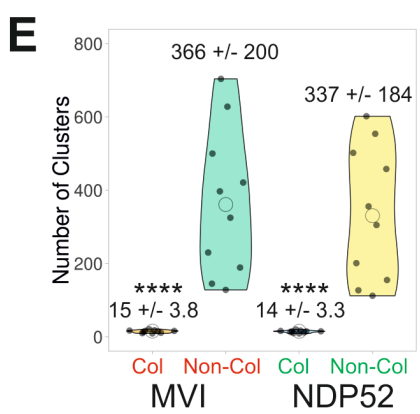


Figure 6: Cluster analysis and colocalization of myosin VI and NDP52

(A) and (B) Two examples of widefield immunofluorescence imaging against endogenous NDP52 (green) and MVI (red) in HeLa cells (Scale bar $10 \mu \mathrm{m}$ ), with their corresponding STORM render. Images were acquired as described in the methods. (C) and (D) Cluster colocalization heatmaps corresponding to the STORM renders shown in (A), depicting the colocalisation scores for each molecular cluster. Here, values of 1 (dark red) correspond to perfectly colocalised clusters and -1 (dark blue) to clusters separated from each other. (E) Results of cluster analysis of MVI and NDP52, displaying the distribution and mean values of the number of clusters per cell ROI, number of molecules per cluster and cluster area. The data are broken down into MVI and NDP52 colocalized and noncolocalized clusters. Individual data points correspond to the corresponding average value per cell ROI. The values represent the mean (+/-s.d.) from the ROIs $(n=10)$ for each condition $\left({ }^{* * *} p<0.0001\right.$ by two-tailed t-test compared to non-colocalized clusters) 
bioRxiv preprint doi: https://doi.org/10.1101/2020.05.10.079236; this version posted May 10, 2020. The copyright holder for this preprint (which was not certified by peer review) is the author/funder, who has granted bioRxiv a license to display the preprint in perpetuity. It is made available under aCC-BY-NC-ND 4.0 International license.

\section{Interactions with binding partners regulates the cellular dynamics of myosin VI.}

Following the single molecule localisation experiments, we performed live cell $3 \mathrm{D}$ single molecule tracking to observe the impact of binding partners on the dynamics of $\mathrm{MVI}$, using an aberration-corrected multi-focal microscope
(acMFM) system [27]. This technique allows the simultaneous acquisition of 9 focal planes covering $4 \mu \mathrm{m}$ in the $z$ axis, with a $20 \times 20 \mu \mathrm{m}$ field of view. We visualised $\mathrm{NI}$ isoform of MVI in HeLa using an N-terminal HaloTag fusion [7] labelled with JF549 [28] and then performed single particle tracking.



B

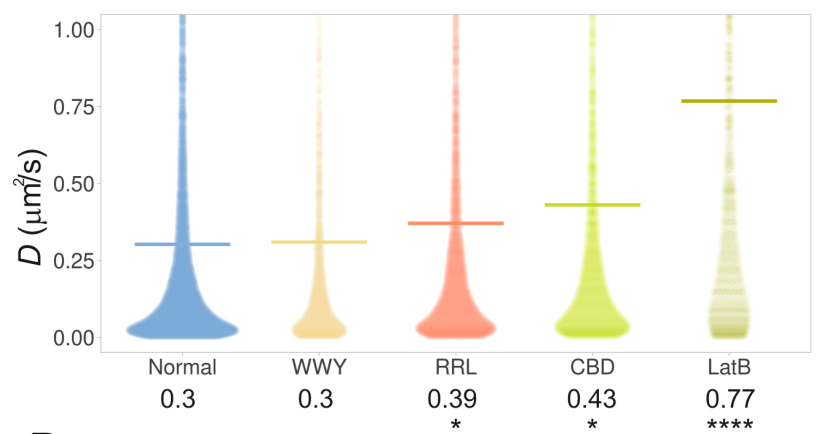

D

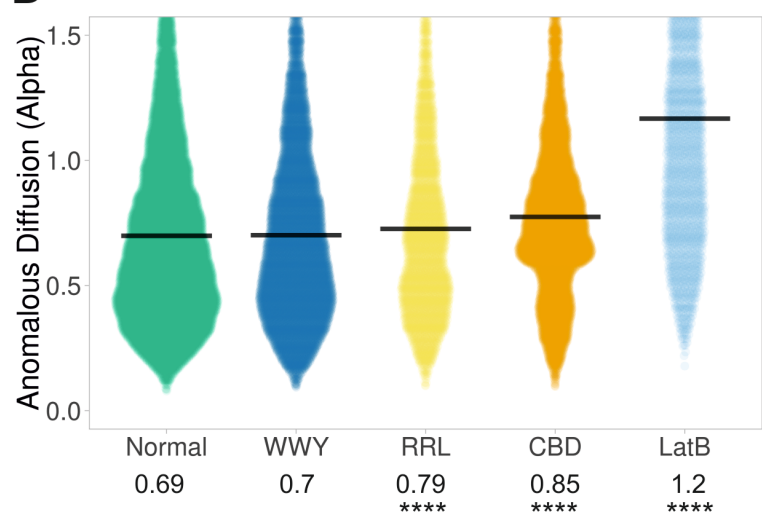

C

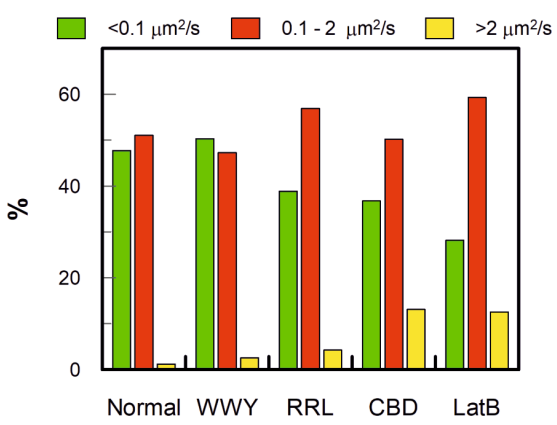

E



Figure 7: Live cell single-molecule dynamics of myosin VI.

(A) Example render of 3D single-molecule trajectories for JF549-labelled Halo-tagged MVI, stably expressed in HeLa cells, under normal conditions. (B) Plot of diffusion constants for wild type (WT) MVI (normal), MVI WWY/WLY (WWY), MVI RRL/AAA (RRL), WT MVI following transient over-expression of GFP-CBD (CBD) and WT MVI following treatment with latrunculin $B$ (LatB). Diffusion constants were derived from fitting trajectories to an anomalous diffusion model, as described in methods. The data represent all trajectories from 100 cells $\left({ }^{*} p<0.05\right.$, ${ }^{* * * *} \mathrm{p}<0.0001$ by two-tailed t-test compared to normal conditions). (C) Diffusion constants $(D)$ were split into three categories corresponding to static/slow moving $\left(D<0.1 \mu \mathrm{m}^{2} \mathrm{~s}^{-1}\right)$, mobile $\left(0.1<D<2 \mu \mathrm{m}^{2} \mathrm{~s}^{-1}\right)$ and hyper-mobile fractions $\left(D>2 \mu \mathrm{m}^{2} \mathrm{~s}^{-1}\right)$. The percentage of molecules from the experiments in $(\mathbf{B})$ falling into each group were then plotted. (D) Plot of anomalous diffusion alpha values derived from the same experimental set shown in (B), for the indicated conditions. ( ${ }^{* * * *} \mathrm{p}<0.0001$ by two-tailed t-test compared to normal conditions). (E) Roseplot representing the angular change in WT MVI diffusion from the trajectories analysed in (B). Angles close to $180^{\circ}$ are enriched which suggests molecules are moving backwards and forwards within a space. 
The trajectories of $\mathrm{MVI}$ revealed different populations of molecules, some undergoing confined motion, some random diffusion and others directed movement (Figure 7A). The diffusion constants (D) extracted from these trajectories had a mean value of $0.3 \mu \mathrm{m}^{2} \mathrm{~s}^{-1}$, under normal conditions, with the large majority of molecules exhibiting slow diffusion (Figure 7B). To better understand the diffusion properties of $\mathrm{MVI}$, we further split these diffusion constants into three groups: a) static/slow moving molecules with $D$ below $0.1 \mu \mathrm{m}^{2} \mathrm{~s}^{-1}$, b) molecules with $D$ between 0.1 and $2 \mu \mathrm{m}^{2} \mathrm{~s}^{-1}$ and c) fast moving molecules with $D$ above $2 \mu \mathrm{m}^{2} \mathrm{~s}^{-1}$ (Figure $7 \mathrm{C}$ ). We found that MVI molecules are almost equally split between the first two categories, indicating that MVI mainly exists in a static/slow and medium diffusive state. In addition, we assessed the type of particle motion exhibited by MVI by calculating the anomalous diffusion alpha value (Figure 7D). Values below 1 are indicative of confined motion, values above 1 are suggestive of directed motion, whereas values of 1 occur for random diffusion. The alpha value for MVI under normal conditions had a mean value of 0.69 , therefore suggesting it mainly undergoes confined movement. This confined motion was further represented using a roseplot (Figure 7E) to depict the angular change in direction within the trajectories. Angles around 180 degrees were enriched and represented trajectories where molecules reversed direction, as would more likely occur for a confined molecule.

To assess the role of binding partners in the cellular dynamics of MVI, we tracked the movement of two MVI mutants in which Dab2 and NDP52 binding was disrupted, namely MVIWWYMLY and MVIRRLAAA, respectively. MVIWWYMLY did not show any significant change in its diffusion properties compared to wild type MVI (Figure 7B and C). This is not surprising since, as shown previously, the $\mathrm{NI}$ isoform displays selectivity for RRL binding partners, rather than the lower affinity WWY-mediated interactions [6]. Conversely, MVIRRL/AAA lead to a significant shift in the distribution of diffusion constants, with a $30 \%$ increase in the mean diffusion constant to a value of $0.39 \mu \mathrm{m}^{2} \mathrm{~s}^{-1}$ (Figure 7B). This can also be seen by the decrease in the static/slow moving population $\left(<0.1 \mu \mathrm{m}^{2} \mathrm{~s}^{-1}\right)$ and the increase in the medium and highly diffusive pools $\left(0.1-2 \mu \mathrm{m}^{2} \mathrm{~s}^{-1}\right.$ and $>2$ $\mu \mathrm{m}^{2} \mathrm{~s}^{-1}$ ) (Figure 7C). This was also matched by the significant change in the type of anomalous diffusion exhibited by the MVI mutant molecules, with an increase in the mean anomalous diffusion alpha value to 0.79 (Figure 7D). As evidenced by the dramatic change in the shape of the distribution, disruption of MVI interaction with its RRL binding partners caused a significant shift from confined motion towards random diffusion.

As expected from the biochemical parameters, these data suggest that there is an impact of the binding partners upon the activity of $\mathrm{MVI}$, which influences its cellular dynamics. This was further supported by the effect of transiently over-expressed GFP-tagged CBD into cells stably expressing wild type Halo-MVI. Overexpression of this construct is known to have a dominant negative effect by displacing wild type MVI from binding partner and lipid-based interactions [29]. Indeed, the presence of the CBD led to a considerable increase in the diffusion constant of $\mathrm{MVI}$ to a mean value of 0.43 $\mu \mathrm{m}^{2} \mathrm{~s}^{-1}$ (Figure 7B), an increase in the population of highly diffusive species (Figure 7C), and a shift of the alpha value to mean of 0.85 (Figure 7D). However, approximately $35 \%$ of MVI molecules still remained static or slow moving, probably reflecting a lack of CBD competition, interactions with actin or non-specific interactions. To assess the contribution of actin to the pool of static/slow diffusing molecules, the dynamics of MVI were observed following treatment with an actin polymerization inhibitor, Latrunculin B. Not surprisingly, there was a significant increase in MVI diffusion (Figure 7B and 7C), due to the loss of actin-based interactions.

Overall, our data demonstrate that the interaction of MVI with its binding partners plays an important role in regulating the cellular dynamics of the protein. The increase in random diffusion observed following disruption of these interactions could reflect a loss of interaction with cargo and anchoring sites. It could also reflect loss of direct interactions with actin, given that $\mathrm{MVI}$ would then be in a back-folded inactive state.

\section{DISCUSSION}

This study has provided novel insights into the mechanisms underlying the regulation of $\mathrm{MVI}$ and its function within a cell. We have revealed that binding partners directly regulate the structural transitions of MVI from an auto-inhibited folded monomer to an unfolded active motor, which is capable of dimerization (Figure 8A). Our data suggest that this mechanism is generic, irrespective of MVI isoform or choice of binding partners. Importantly, this model does not assume that binding partners must be dimeric. Moreover, pre-steady-state kinetics has allowed us to define that binding partners first bind to the backfolded $\mathrm{MVI}$ and subsequently trigger unfolding, rather than binding to a spontaneously unfolded MVI and stabilising that state. 


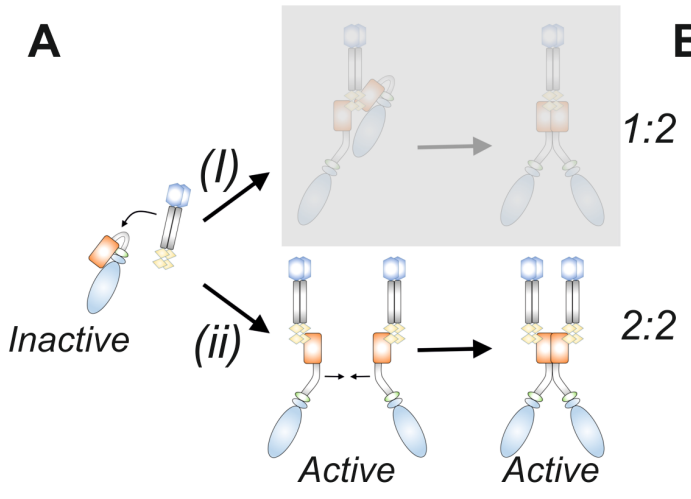

B

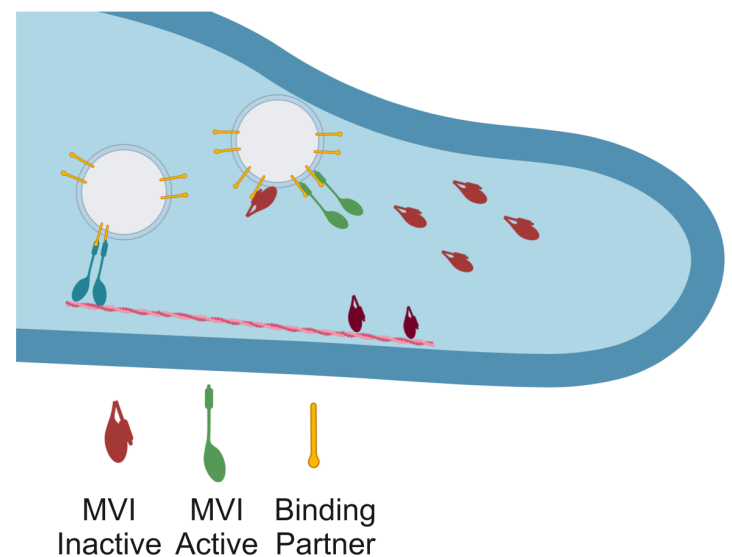

Figure 8: Model describing the activation of myosin VI by binding partners.

(A) Two routes of binding partner-dependent dimerization of MVI. (i) Dimeric binding partner (e.g. NDP52) binds to backfolded MVI and triggers its unfolding. A second MVI molecule is then recruited through the binding partner, due to its dimeric nature. This results in a stoichiometry of 1 dimeric partner to $2 \mathrm{MVI}$ motors. This mechanism does not require MVI internal dimerization. (ii) Each MVI molecule is unfolded by an individual binding partner. Unfolding then exposes internal dimerization sequences within MVI, which can result in a dimer complex with a stoichiometry of 2 partners and 2 motors. The data presented here allow option (i) to be excluded. Our data also allows us to conclude that the folded state is inactive (non-actin bound) and that the unfolded state (monomer and dimer) becomes active with the ability to bind actin. (B) Recruitment and activation of myosin VI to cellular cargo by binding partners. Monomeric backfolded (inactive) myosin VI (red) can be found freely diffusing in the cytoplasm or bound to actin (dark red). The inactive myosin VI associates with cargo-bound binding partners. This triggers unfolding and activation of the motor in monomeric (green) or dimeric (blue) populations. The active myosin VI can interact with actin filaments and undergo processive movement to facilitate cargo transportation.

We have shown that once MVI is unfolded, it has the intrinsic ability to dimerize, as it has been reported before $[4,6,13,23,24,30]$. Here, we have further clarified these models by showing that MVI dimerizes internally and that binding partner dimerization is not the driving factor. We therefore propose that the complex stoichiometry exists as one active binding partner (monomer or dimer) per MVI molecule (Figure 8). This stoichiometry has been reported from structural studies [24, 31]. Rapid mixing kinetics allowed us to probe the individual mechanistic steps and therefore propose that dimerization can occur rapidly following unfolding of $\mathrm{MVI}$, with unfolding being the rate-limiting step once binding partner interaction occurs.

MVI exists as four alterative spliced isoforms and interacts with a wide range of binding partners. In our study, we assessed the generic nature of $\mathrm{MVI}$ unfolding and dimerization, using examples of two MVI isoforms, which represent the two largest structural changes, and two binding partners, which bind at alternative motifs. Although we cannot exclude potential differences in behaviour in other isoforms and with other MVI partners, this is quite unlikely. We draw this conclusion because all known binding partners associate with one of two sites on MVI, as depicted here, and we propose that interactions at these sites trigger MVI unfolding. Greater differences in oligomerization status may occur between binding partners where monomers or dimers can be favoured. Moreover, larger oligomers, while maintaining 1:1 stoichiometry, could also be produced [31].

Our biochemical analysis has furthered our understanding of how MVI interacts with its binding partners and highlighted the impact of these interactions upon MVI activity. We have previously shown that this relies on the interplay between isoform splicing and the differential affinities between the RRL and WWY binding sites motifs [6]. Here, we have provided insights into the order of events occurring during partner binding, unfolding and dimerization. However, by nature, all these biochemical studies occur under an environment allowing free molecular diffusion, which is strikingly different from the crowded cellular environment. Our high-resolution imaging data shed light on how the biochemical features of MVI measured in vitro could translate into the complex cellular context. They revealed that over $50 \%$ of MVI molecules are clustered within the mammalian cell. The high local densities within these clusters enhance the probability of interactions with its binding partners, which have been biochemically shown to be in the micromolar range. Moreover, our data have shown that association with binding partners further increases these clusters in size and molecular density. Although this was tested here only for the partner NDP52, it is very likely to apply for all binding partners. The reason is that, within such clusters, multivalent interactions of 
$\mathrm{MVI}$ to cargo through various binding partners would lead to stable complexes capable of longrange processive movement. Enzymatic clustering has been reported for many cellular processes [32, 33] where is it hypothesised to increase local concentrations to enhance efficiency. Therefore, given the multifunctional and complex nature of MVI intracellular activity, it would not be surprising that this myosin follows a mechanism, which allows stability, efficiency and high level of regulation.

In addition to what was demonstrated biochemically, the impact of binding partners upon MVI is also evident from the live cell singlemolecule tracking data. Here, we have shown how mutations, or truncations that lead to the disruption of interactions between $\mathrm{MVI}$ and binding partners have a profound effect on the dynamics of the protein inside the cell, leading to loss of the static and slow-moving molecules, which display restrictive diffusion, for more rapidly diffusing molecules exhibiting random motion. To account for such a behavioural switch, we propose that the direct association with the actin cytoskeleton and cellular cargo is decreased when interactions with binding partners are disrupted. Indeed, disruption of the actin cytoskeleton by latrunculin B treatment further drives MVI into a rapid freely diffusive state. However, if cargo interactions are maintained, such as through direct lipid binding, then molecules would not diffuse freely. Likewise, MVI molecules may back-fold on actin and remain in an off-state. Due to these possibilities and the crowded cellular environment, we did not observe a total loss of static molecules.

These observations allowed us to further expand our activation model of MVI. We propose that the back-folded MVI, being inactive, remains stationary on actin or unbound and randomly diffusing in the cell. In this latter state, MVI can be sequestered by binding partners to cellular cargo or organelles, and get subsequently activated (Figure 8B). Diffusion of MVI in its inactive state is important because it allows sampling of various regions of the cell before recruitment by its partners to function in specific biological processes. Interestingly, we would expect to observe a decrease in ATPase activity if the protein is not bound to actin. However, these changes have not been observed through biochemical analysis, possibly due to the marked differences between the chemical environment of biochemical assays and the cellular environment. Also, within the complex cellular environment, other processes, such as further regulation of the motor by calcium-calmodulin or changes in the phosphorylation status of MVI upon perturbation of binding partner interactions, could lead to inhibition of the ATPase activity, and these would not be observed in vitro. These factors remain to be determined.

In summary, our multidisciplinary study proposes a generic mechanism for the regulation of MVI by binding partners in the cell. It remains unknown as to how monomeric or dimeric states of the protein are selected within the cell, which may be the role of the binding partners to fulfil. Interestingly, due to the internal dimerization of MVI, we suggest that monomeric activities would arise from binding partners activity halting dimerization. Given the established impact of MVI in several diseases, including cancer [19, 34-37] and deafness [38,39], defining the mechanistic details of its regulation and function is critical for understanding the impact of mutations, truncations, or altered expression of $\mathrm{MVI}$ or partners during disease. Overall, these insights provide new avenues for exploring how the activity of this multi-functional motor protein is regulated within the cell and how these processes may be perturbed during disease.

\section{ACKNOWLEDGEMENTS}

We thank the UKRI-MRC (MR/M020606/1) and UKRISTFC (19130001) for funding. Aberration-corrected multi-focal microscopy was performed in collaboration with the Advanced Imaging Center at Janelia Research Campus, a facility jointly supported by the Howard Hughes Medical Institute and the Gordon and Betty Moore Foundation. We also thank Darren Griffin (University of Kent) and Mike Geeves (University of Kent) for sharing of equipment and reagents, and Satya Khuon (Janelia Research Campus) for assisting with cell culture. The JF549 dye was kindly provided by Luke Lavis (Janelia Research Campus).

\section{AUTHOR CONTRIBUTIONS}

C.P.T. conceived the study. A.dS., N.F., Y.H-G. and C.P.T. designed experiments. N.F. and C.P.T. designed and cloned constructs. A.dS., N.F., Y.H-G., R.E.G. and C.P.T. performed single molecule imaging experiments. Imaging was supported by L.W., M.M-F. and J.A. N.F., R.E.G. and C.P.T. expressed, purified and performed experiments with recombinant proteins. L.W., J.A., T-L.C., A.dS. and C.P.T. contributed to single molecule data analysis. C.P.T. supervised the study. C.P.T. wrote the manuscript with comments from all authors.

Competing financial interests: The authors declare no competing financial interests.

\section{METHODS}

\section{Constructs}

A list of constructs and PCR primers are provided in Supplementary Table 1 and 2 , respectively. Constructs generated in this work are described below: RRL/AAA and WWY/WLY mutations were made by site-directed mutagenesis using standard Quick-Change sitedirected mutagenesis protocol with pLV-Tet0-Halo MVI as the template. All plasmids were verified by DNA sequencing. 
bioRxiv preprint doi: https://doi.org/10.1101/2020.05.10.079236; this version posted May 10, 2020. The copyright holder for this preprint (which was not certified by peer review) is the author/funder, who has granted bioRxiv a license to display the preprint in perpetuity. It is made available under aCC-BY-NC-ND 4.0 International license.

\section{Protein expression and purification in Escherichia coli}

Recombinant constructs were expressed in E.coli BL21 DE3 cells (Invitrogen) in Luria Bertani media. Proteins were purified by affinity chromatography (HisTrap FF, GE Healthcare). The purest fractions were desalted through a PD10 column (GE Healthcare) to remove imidazole before treatment with TEV protease for $4 \mathrm{hr}$ at $25^{\circ} \mathrm{C}$. The samples were then passed through a second HisTrap column. The cleaved protein was further purified through a Superdex 200 16/600 column (GE Healthcare).

\section{Protein Expression using Baculovirus system}

Full-length MVI NI, MVI ${ }_{1-814}, \mathrm{MVI}_{1-1060}$ and Xenopus calmodulin were expressed in Sf9 and Sf21 (Spodoptera frugiperda) insect cells using the Bac-toBac® Baculovirus Expression System (Invitrogen). Sf9 cells were cultured in Sf900 media (Gibco). Recombinant bacmids were generated following the manufacturer's instructions and were transfected into adherent Sf9 cells to generate the P1 viral stock. Sf9 cells were infected in suspension at $27^{\circ} \mathrm{C}$ and $100 \mathrm{rpm}$ with 1 in 50 dilution of $\mathrm{P} 1$ and $\mathrm{P} 2$ viral stocks to yield $\mathrm{P} 2$ and $\mathrm{P} 3$ stocks, respectively. Finally, expression of recombinant proteins was set up by infecting sf21 cells with the P3 viral stock in Spodopan media (PAN Biotech). To ensure correct folding of the MVI constructs, cells were simultaneously infected with P3 viral stock of the $\mathrm{MVI}$ constructs together with calmodulin at a 0.75 ratio. The cells were harvested after 3 days by centrifugation for $15 \mathrm{~min}$ at $700 \mathrm{xg}$ and at $4{ }^{\circ} \mathrm{C}$ and resuspended in ice cold myosin extraction buffer $\left(90 \mathrm{mM} \mathrm{KH}_{2} \mathrm{PO}_{4}, 60 \mathrm{mM} \mathrm{K}_{2} \mathrm{HPO}_{4}, 300 \mathrm{mM} \mathrm{KCl}\right.$, $\mathrm{pH}$ 6.8), supplemented with Proteoloc protease inhibitor cocktail (Expedeon) and $100 \mu \mathrm{M}$ PMSF, before proceeding to protein purification. Prior to sonication, an additional $5 \mathrm{mg}$ recombinant calmodulin was added together with $2 \mathrm{mM}$ DTT. After sonication, $5 \mathrm{mM} \mathrm{ATP}$ and $10 \mathrm{mM} \mathrm{MgCl}_{2}$ were added and the solution was rotated at $4{ }^{\circ} \mathrm{C}$ for $30 \mathrm{~min}$ before centrifugation $\left(20,000 \mathrm{~g}, 4^{\circ} \mathrm{C}, 30 \mathrm{~min}\right)$. Then, the cell lysate was subjected to the purification. Proteins were purified by affinity chromatography (HisTrap FF, GE Healthcare). The purest fractions were further purified through a Superdex $200 \quad 16 / 600$ column (GE Healthcare).

\section{Protein labelling}

Proteins were transferred into $50 \mathrm{mM}$ Na-phosphate $(\mathrm{pH}$ 6.5) using a PD10 desalting column. Samples were then incubated with a 5-fold excess of dye for 4 hours, rotating at $4^{\circ} \mathrm{C}$. Excess dye was removed using a PD10 desalting column pre-equilibrated with $50 \mathrm{mM}$ Na-Phosphate, $150 \mathrm{mM} \mathrm{NaCl}$ and $1 \mathrm{mM}$ DTT. Labelling efficiency was calculated based on the absorbance at $280 \mathrm{~nm}$ and the absorbance maximum of the dye. Typical efficiency was $90 \%$, whereby the less than complete labelling was taken as an indicator for a single dye per protein. This was tested for isolated preparations in mass spectroscopy, which revealed both an unlabelled and single labelled population.

\section{Cell culture and Transfection}

HeLa (ECACC 93021013) cells were cultured at $37^{\circ} \mathrm{C}$ and $5 \% \mathrm{CO}_{2}$, in Gibco MEM Alpha medium with
GlutaMAX (no nucleosides), supplemented with $10 \%$ Fetal Bovine Serum (Gibco), 100 units/ml penicillin and $100 \mu \mathrm{g} / \mathrm{ml}$ streptomycin (Gibco). For the transient expression of MVI mutants, HeLa cells grown on glass coverslips were transfected using Lipofectamine 2000 (Invitrogen), following the manufacturer's instructions. Depending on the construct, $24 \mathrm{~h}-72 \mathrm{~h}$ after transfection, cells were subjected to further analysis. To inhibit actin polymerization, cells were treated with $1 \mu \mathrm{M}$ Latrunculin B (Sigma) for $1 \mathrm{~h}$ at $37^{\circ} \mathrm{C}$.

\section{Immunofluorescence}

HeLa cells were fixed for $15 \mathrm{~min}$ at room temperature in $4 \%(\mathrm{w} / \mathrm{v})$ paraformaldehyde (PFA) in PBS and residual PFA was quenched for 15 min with $50 \mathrm{mM}$ ammonium chloride in PBS. All subsequent steps were performed at room temperature. Cells were permeabilised and simultaneously blocked for $15 \mathrm{~min}$ with $0.1 \%(\mathrm{v} / \mathrm{v})$ Triton $\mathrm{X}-100$ and $2 \%(\mathrm{w} / \mathrm{v})$ BSA in PBS. Cells were then immuno-stained against the endogenous proteins by $1 \mathrm{~h}$ incubation with the indicated primary and subsequently the appropriate fluorophore-conjugated secondary antibody (details below), both diluted in $2 \%(\mathrm{w} / \mathrm{v})$ BSA in PBS. The following antibodies were used at the indicated dilutions: Rabbit anti-myosin VI (1:200, Atlas-Sigma HPA0354863), Mouse anti-NDP52 (1:250 Abcam ab124372), Donkey anti-mouse Alexa Fluor 488conjugated (1:250, Abcam Ab181289), Donkey antirabbit Alexa Fluor 647-conjugated (1:250, Abcam Ab181347). Coverslips were mounted on microscope slides with Mowiol (10\% (w/v) Mowiol 4-88, 25\% (w/v) glycerol, $0.2 \mathrm{M}$ Tris- $\mathrm{HCl}, \mathrm{pH} 8.5$ ), supplemented with $2.5 \%(\mathrm{w} / \mathrm{v})$ of the anti-fading reagent DABCO (Sigma).

\section{STORM Imaging}

Cells were seeded on pre-cleaned No 1.5, 25-mm round glass coverslips, placed in 6 -well cell culture dishes. Glass coverslips were cleaned by incubating them for 3 hours, in etch solution, made of 5:1:1 ratio of $\mathrm{H}_{2} \mathrm{O}: \mathrm{H}_{2} \mathrm{O}_{2}$ (50 wt. \% in $\mathrm{H}_{2} \mathrm{O}$, stabilized, Fisher Scientific) : $\mathrm{NH}_{4} \mathrm{OH}$ (ACS reagent, 28-30\% $\mathrm{NH}_{3}$ basis, Sigma), placed in a $70^{\circ} \mathrm{C}$ water bath. Cleaned coverslips were repeatedly washed in filtered water and then ethanol, dried and used for cell seeding. Cells were fixed in pre-warmed 4\% (w/v) PFA in PBS and residual PFA was quenched for 15 min with $50 \mathrm{mM}$ ammonium chloride in PBS. Immunofluorescence (IF) was performed in filtered sterilised PBS. Cells were permeabilized and simultaneously blocked for $30 \mathrm{~min}$ with $3 \%(\mathrm{w} / \mathrm{v})$ BSA in PBS supplemented with $0.1 \%$ (v/v) Triton X-100. Permeabilized cells were incubated for $1 \mathrm{~h}$ with the primary antibody and subsequently the appropriate fluorophore-conjugated secondary antibody, at the desired dilution in 3\% (w/v) BSA, $0.1 \%$ $(\mathrm{v} / \mathrm{v})$ Triton X-100 in PBS. The antibody dilutions used were the same as for the normal IF protocol (see above). Following incubation with both primary and secondary antibodies, cells were washed 3 times, for 10 min per wash, with $0.2 \%(\mathrm{w} / \mathrm{v}) \mathrm{BSA}, 0.05 \%(\mathrm{v} / \mathrm{v})$ Triton X-100 in PBS or TBS. Cells were further washed in PBS and fixed for a second time with pre-warmed $4 \%(\mathrm{w} / \mathrm{v})$ PFA in PBS for $10 \mathrm{~min}$. Cells were washed in PBS and stored at $4{ }^{\circ} \mathrm{C}$, in the dark, in $0.02 \% \mathrm{NaN}_{3}$ in PBS, before proceeding to STORM imaging.

Before imaging, coverslips were assembled into the Attofluor ${ }^{\circledR}$ cell chambers (Invitrogen). Imaging was 
bioRxiv preprint doi: https://doi.org/10.1101/2020.05.10.079236; this version posted May 10, 2020. The copyright holder for this preprint (which was not certified by peer review) is the author/funder, who has granted bioRxiv a license to display the preprint in perpetuity. It is made available under aCC-BY-NC-ND 4.0 International license.

performed in freshly made STORM buffer consisting of $10 \%$ (w/v) glucose, $10 \mathrm{mM} \mathrm{NaCl}, 50 \mathrm{mM}$ Tris - $\mathrm{pH}$ 8.0, supplemented with $0.1 \%(\mathrm{v} / \mathrm{v})$ 2-mercaptoethanol and $0.1 \%(\mathrm{v} / \mathrm{v})$ pre-made GLOX solution which was stored at $4{ }^{\circ} \mathrm{C}$ for up to a week $(5.6 \%(\mathrm{w} / \mathrm{v})$ glucose oxidase and $3.4 \mathrm{mg} / \mathrm{ml}$ catalase in $50 \mathrm{mM} \mathrm{NaCl}, 10 \mathrm{mM}$ Tris $\mathrm{pH}$ 8.0). All chemicals were purchased from Sigma. Imaging was undertaken using the Zeiss Elyra PS.1 system. Illumination was from a HR Diode $642 \mathrm{~nm}(150$ $\mathrm{mW})$ and HR Diode $488 \mathrm{~nm}(100 \mathrm{~mW})$ lasers where power density on the sample was $7-14 \mathrm{~kW} / \mathrm{cm}^{2}$ and 7 $12 \mathrm{~kW} / \mathrm{cm}^{2}$, respectively

Imaging was performed under highly inclined and laminated optical (HILO) illumination to reduce the background fluorescence with a 100x NA 1.46 oil immersion objective lens (Zeiss alpha PlanApochromat) with a BP 420-480/BP495-550/LP 650 filter. The final image was projected on an Andor iXon EMCCD camera with $25 \mathrm{msec}$ exposure for 20000 frames.

Image processing was performed using the Zeiss Zen software. Where required, two channel images were aligned following a calibration using a calibration using pre-mounted MultiSpec bead sample (Carl Zeiss, 2076-515). The channel alignment was then performed in the Zeiss Zen software using the Affine method to account for lateral, tilting and stretching between the channels. The calibration was performed during each day of measurements.

The images were then processed through our STORM analysis pipeline using the Zen software. Single molecule detection and localisation was performed using a 9 pixel mask with a signal to noise ratio of 6 in the "Peak finder" settings while applying the "Account for overlap" function. This function allows multi-object fitting to localise molecules within a dense environment. Molecules were then localised by fitting to a 2D Gaussian.

The render was then subjected to model-based crosscorrelation drift correction. Typical localisation precision was $20 \mathrm{~nm}$ for Alexa-Fluor 647 and $30 \mathrm{~nm}$ for Alexa-Fluor 488. The final render was then generated at $10 \mathrm{~nm} /$ pixel and displayed in Gauss mode where each localisation is presented as a $2 \mathrm{D}$ gaussian with a standard deviation based on its precision. The localisation table was exported as a txt for import in to Clus-DoC

\section{Clus-DoC}

The single molecule positions were exported from Zeiss black version and imported into the Clus-DoC analysis software

\section{(https://github.com/PRNicovich/ClusDoC).}

Cytoplasmic areas were selected as ROls for cluster analysis. First the Ripley $\mathrm{K}$ function was completed on each channel identifying the $r$ max. The $r$ max was then assigned for DBSCAN if one channel was being analysed or Clus-Doc if two channel colcalisation was being analysed. The clustering size was set to a minimum of 5 molecules, with smoothing set at $7 \mathrm{~nm}$ and epsilon set at the mean localization precision for the dye. All other analyses parameters remained at default settings. Data concerning each cluster was exported and graphed using Plots of Data.

\section{Size-exclusion Chromatography and Multi-Angle Light Scattering}

$100 \mu \mathrm{l}$ samples of $2 \mathrm{mg} / \mathrm{ml}$ purified protein, was applied to a Superdex $200(30 \times 1 \mathrm{~cm})$ analytical column (GE Healthcare) equilibrated in $150 \mathrm{mM} \mathrm{NaCl}, 50 \mathrm{mM}$ Tris. $\mathrm{HCl}(\mathrm{pH} 7.5)$ and $1 \mathrm{mM}$ DTT and controlled using Waters 626 HPLC at room temperature. Eluted proteins were analysed with Viscotek SEC-MALS 9 and Viscotek RI detector VE3580 (Malvern Panalytical). Molecular mass was determined using OmniSEC software.

Multi-focal Imaging and Particle Tracking Analysis Cells stably or transiently expressing Halo-tag constructs were labelled for $15 \mathrm{~min}$ with $10 \mathrm{nM}$ HaloTag-JF549 ligand, in cell culture medium at $37^{\circ} \mathrm{C}$, $5 \% \mathrm{CO}_{2}$. Cells were washed for 3 times with warm cell culture medium and then incubated for further $30 \mathrm{~min}$ at $37^{\circ} \mathrm{C}, 5 \% \mathrm{CO}_{2}$. Cells were then washed three times in pre-warmed FluoroBrite DMEM imaging medium (ThermoFisher Scientific), before proceeding to imaging.

Single molecule imaging was performed using an aberration-corrected multifocal microscope (acMFM), as described by Abrahamsson et al. [27]. Briefly, samples were imaged using $561 \mathrm{~nm}$ laser excitation, with typical irradiance of $4-6 \mathrm{~kW} / \mathrm{cm}^{2}$ at the back aperture of a Nikon 100x 1.4 NA objective. Images were relayed through a custom optical system appended to the detection path of a Nikon Ti microscope with focus stabilization. The acMFM detection path includes a diffractive multifocal grating in a conjugate pupil plane, a chromatic correction grating to reverse the effects of spectral dispersion, and a nine-faceted prism, followed by a final imaging lens.

The acMFM produces nine simultaneous, separated images, each representing successive focal planes in the sample, with ca. $20 \mu \mathrm{m}$ field of view and nominal axial separation of ca. $400 \mathrm{~nm}$ between them. The nine-image array is digitized via an electron multiplying charge coupled device (EMCCD) camera (iXon Du897, Andor) at up to $32 \mathrm{~ms}$ temporal resolution, with typical durations of 30 seconds.

$3 D+t$ images of single molecules were reconstructed via a calibration procedure, implemented in Matlab (MathWorks), that calculates and accounts for (1) the inter-plane spacing, (2) affine transformation to correctly align each focal plane in the xy plane with respect to each other, and (3) slight variations in detection efficiency in each plane, typically less than $\pm 5-15 \%$ from the mean.

Reconstructed data were then subject to preprocessing, including background subtraction, mild deconvolution (3-5 Richardson-Lucy iterations), and/or Gaussian de-noising prior to 3D particle tracking using the MOSAIC software suite [40]. Parameters were set where maximum particle displacement was $400 \mathrm{~nm}$ and a minimum of 10 frames was required. Tracks were reconstructed, and diffusion constants were extracted via MSD analysis [41] using custom Matlab software assuming an anomalous diffusion model.

\section{Steady-state ATPase Activity of MVI}

$\mathrm{Ca}^{2+}$-actin monomers were converted to $\mathrm{Mg}^{2+}$-actin with $0.2 \mathrm{mM}$ EGTA and $50 \mu \mathrm{M} \mathrm{MgCl} 2$ before polymerizing by dialysis into $20 \mathrm{mM}$ Tris. $\mathrm{HCl}(\mathrm{pH} 7.5)$, $20 \mathrm{mM}$ imidazole $(\mathrm{pH} 7.4), 25 \mathrm{mM} \mathrm{NaCl}$ and $1 \mathrm{mM}$ DTT. A 1.1 molar equivalent of phalloidin (Sigma) was 
bioRxiv preprint doi: https://doi.org/10.1101/2020.05.10.079236; this version posted May 10, 2020. The copyright holder for this preprint (which was not certified by peer review) is the author/funder, who has granted bioRxiv a license to display the preprint in perpetuity. It is made available under aCC-BY-NC-ND 4.0 International license.

used to stabilize actin filaments, as previously described [42].

Steady-state ATPase activities were measured at 25 ${ }^{\circ} \mathrm{C}$ in KMg50 buffer (50

$\mathrm{mM} \mathrm{KCl}, 1 \mathrm{mM} \mathrm{MgCl} 2,1 \mathrm{mM}$ EGTA, $1 \mathrm{mM}$ DTT, and $10 \mathrm{mM}$ imidazole, $\mathrm{pH}$ 7.0). Supplemented with the $\mathrm{NADH}$-coupled assay components, $0.2 \mathrm{mM} \mathrm{NADH}, 2$ $\mathrm{mM}$ phosphoenolpyruvate, $3.3 \mathrm{U} \mathrm{\textrm {ml } ^ { - 1 }}$ lactate dehydrogenase, $2.3 \mathrm{U} \mathrm{ml}^{-1}$ pyruvate kinase and various actin concentrations $(0-30 \mu \mathrm{M})$. The final [Mg.ATP] was $5 \mathrm{mM}$ and MVI concentration was 100 $300 \mathrm{nM}$. The assay was started by the addition of MVI. The change in absorption at $\mathrm{OD}_{340} \mathrm{~nm}$ was followed for $5 \mathrm{~min}$. The $k_{\text {cat }}$ and $K_{\text {actin }}$ values were determined by fitting the data to equation 1.

$$
\text { Rate }=V_{\mathrm{o}}+\left(\frac{k_{\mathrm{cat}}[\text { Actin }]}{K_{\mathrm{actin}}+[\text { Actin }]}\right)
$$

$V_{0}$ is the basal ATPase activity of MVI, $k_{\text {cat }}$ is the maximum actin-activated ATPase rate and $K_{\text {actin }}$ is the concentration of actin needed to reach half maximal ATPase activity.

\section{Titration measurements}

All reactions were performed at $25{ }^{\circ} \mathrm{C}$ in a buffer containing $50 \mathrm{mM}$ Tris. $\mathrm{HCl}(\mathrm{pH} 7.5), 150 \mathrm{mM}$ sodium chloride and $1 \mathrm{mM}$ DTT in a final volume of $100 \mu \mathrm{L}$. Measurements were performed using a ClarioStar Plate Reader (BMG Labtech). Intensity measurements were performed at the following wavelengths: FITC (ex. 490nm), Alexa Fluor 555 (ex. 555nm). FITC to Alexa Fluor 555 FRET measurements were performed using the following wavelengths ex. $470 \mathrm{~nm}$ and em. $575 \mathrm{~nm}$.

\section{Stopped flow measurements}

A HiTech SF61DX2 apparatus (TgK Scientific Ltd, Bradford-on-Avon, UK) with a mercury-xenon light source and HiTech Kinetic Studio 2 software was used $[43,44]$. For FRET experiments, excitation was at 495 $\mathrm{nm}$ with emission through a $570 \mathrm{~nm}$ cut-off filter (Schott Glass). For Cy3B, excitation was at $550 \mathrm{~nm}$ with emission through a $570 \mathrm{~nm}$ cut-filter (Schott Glass). In all experiments, the quoted concentrations are those in the mixing chamber, except when stated. All experiments were performed at $25^{\circ} \mathrm{C}$ in $50 \mathrm{mM}$ Tris$\mathrm{HCl}, 150 \mathrm{mM} \mathrm{NaCl}, 1 \mathrm{mM}$ DTT and $3 \mathrm{mM} \mathrm{MgCl}_{2}$. The dead time of the stopped-flow instrument was $\sim 2 \mathrm{~ms}$ : during this initial time no change in fluorescence can be observed.

\section{Analysis of kinetic data}

For the FRET titrations: The $575 \mathrm{~nm}$ intensity data was corrected for the increase in intensity due to a small direct excitation. This background signal was subtracted from the dataset to leave the FRET values. The titration curves for the $\mathrm{MVI}_{\text {TAlL }}$ interactions were fitting to a binding quadratic equation, Equation 2:

[2]

$$
=\frac{\left([\text { FITC }]_{t}+[\text { AF555 }]_{t}+K_{d}\right)-\sqrt{\left([\text { [FITC }]_{t}+[A F 555]_{t}+K_{d}\right)^{2}-4[\text { [FITC }]_{t}[A F 555]_{t}}}{2}
$$

\section{Graphics}

Unless stated, data fitting and plotting was performed using Plots of data [45] and Grafit Version 5 (Erithacus
Software Ltd). Cartoons were generated using the BioRender software.

\section{Data Availability}

The data supporting the findings of this study are available from the corresponding author on request.

\section{REFERENCES}

1. Fili, N. and C.P. Toseland, Unconventional Myosins: How Regulation Meets Function. Int J Mol Sci, 2019. 21(1).

2. Roberts, R., et al., Myosin VI: cellular functions and motor properties. Philos Trans R Soc Lond B Biol Sci, 2004. 359(1452): p. 1931-44.

3. Vreugde, S., et al., Nuclear myosin VI enhances RNA polymerase II-dependent transcription. Mol Cell, 2006. 23(5): p. 749-55.

4. Fili, N., et al., NDP52 activates nuclear myosin VI to enhance RNA polymerase II transcription. Nat Commun, 2017. 8(1): p. 1871

5. Cook, A., Y. Hari-Gupta, and C.P. Toseland, Application of the SSB biosensor to study in vitro transcription. Biochem Biophys Res Commun, 2018. 496(3): p. 820-825.

6. Fili, N., et al., Competition between two high-and low-affinity protein-binding sites in myosin $\mathrm{VI}$ controls its cellular function. J Biol Chem, 2020. 295(2): p. 337-347.

7. Große-Berkenbusch, A., et al., Myosin VI moves on nuclear actin filaments and supports longrange chromatin rearrangements. bioRxiv, 2020: p. 2020.04.03.023614.

8. Spudich, G., et al., Myosin VI targeting to clathrincoated structures and dimerization is mediated by binding to Disabled-2 and PtdIns(4,5)P2. Nat Cell Biol, 2007. 9(2): p. 176-83.

9. Hari-Gupta, Y., et al., Nuclear myosin VI regulates the spatial organization of mammalian transcription initiation. bioRxiv, 2020: p. 2020.04.21.053124.

10. Wells, A.L., et al., Myosin VI is an actin-based motor that moves backwards. Nature, 1999. 401(6752): p. 505-8.

11. Buss, F., G. Spudich, and J. Kendrick-Jones, Myosin VI: cellular functions and motor properties. Annu Rev Cell Dev Biol, 2004. 20: p. 649-76.

12. Finan, D., M.A. Hartman, and J.A. Spudich, Proteomics approach to study the functions of Drosophila myosin VI through identification of multiple cargo-binding proteins. Proc Natl Acad Sci U S A, 2011. 108(14): p. 5566-71.

13. Mukherjea, M., et al., Myosin VI dimerization triggers an unfolding of a three-helix bundle in order to extend its reach. Mol Cell, 2009. 35(3): p. 305-15.

14. Knight, P.J., et al., The predicted coiled-coil domain of myosin 10 forms a novel elongated domain that lengthens the head. J Biol Chem, 2005. 280(41): p. 34702-8.

15. Buss, F., et al., Myosin VI isoform localized to clathrin-coated vesicles with a role in clathrin- 
bioRxiv preprint doi: https://doi.org/10.1101/2020.05.10.079236; this version posted May 10, 2020. The copyright holder for this preprint (which was not certified by peer review) is the author/funder, who has granted bioRxiv a license to display the preprint in perpetuity. It is made available under aCC-BY-NC-ND 4.0 International license.

mediated endocytosis. EMBO J, 2001. 20(14): p. 3676-84.

16. $\quad \mathrm{Au}, \mathrm{J} . \mathrm{S}$., et al., Myosin VI is required for sorting of AP-1B-dependent cargo to the basolateral domain in polarized MDCK cells. J Cell Biol, 2007. 177(1): p. 103-14.

17. Morriswood, B., et al., T6BP and NDP52 are myosin VI binding partners with potential roles in cytokine signalling and cell adhesion. J Cell Sci, 2007. 120(Pt 15): p. 2574-85.

18. Naccache, S.N., T. Hasson, and A. Horowitz, Binding of internalized receptors to the PDZ domain of GIPC/synectin recruits myosin $\mathrm{VI}$ to endocytic vesicles. Proc Natl Acad Sci U S A, 2006. 103(34): p. 12735-40.

19. Wollscheid, H.P., et al., Diverse functions of myosin VI elucidated by an isoform-specific alphahelix domain. Nat Struct Mol Biol, 2016. 23(4): p. 300-8.

20. Toseland, C.P., Fluorescent labeling and modification of proteins. J Chem Biol, 2013. 6(3): p. 85-95.

21. Batters, C., et al., Calcium can mobilize and activate myosin-VI. Proc Natl Acad Sci U S A, 2016. 113(9): p. E1162-9.

22. Mukherjea, M., et al., Myosin VI must dimerize and deploy its unusual lever arm in order to perform its cellular roles. Cell Rep, 2014. 8(5): p. 1522-32.

23. Phichith, D., et al., Cargo binding induces dimerization of myosin VI. Proc Natl Acad Sci U S A, 2009. 106(41): p. 17320-4.

24. $\mathrm{Yu}, \mathrm{C}$. , et al., Myosin VI undergoes cargomediated dimerization. Cell, 2009. 138(3): p. 53748.

25. Altman, D., H.L. Sweeney, and J.A. Spudich, The mechanism of myosin VI translocation and its load-induced anchoring. Cell, 2004. 116(5): p. 737-49.

26. Pageon, S.V., et al., Clus-DoC: a combined cluster detection and colocalization analysis for singlemolecule localization microscopy data. Mol Biol Cell, 2016. 27(22): p. 3627-3636.

27. Abrahamsson, S., et al., Fast multicolor 3D imaging using aberration-corrected multifocus microscopy. Nat Methods, 2013. 10(1): p. 60-3.

28. Grimm, J.B., et al., Bright photoactivatable fluorophores for single-molecule imaging. Nat Methods, 2016. 13(12): p. 985-988.

29. Bond, L.M., et al., Myosin VI and its binding partner optineurin are involved in secretory vesicle fusion at the plasma membrane. Mol Biol Cell, 2011. 22(1): p. 54-65.

30. Park, H., et al., Full-length myosin VI dimerizes and moves processively along actin filaments upon monomer clustering. Mol Cell, 2006. 21(3): p. 331-6.

31. Shang, G., et al., Structure analyses reveal a regulated oligomerization mechanism of the PlexinD1/GIPC/myosin VI complex. Elife, 2017. 6.

32. Carmo-Fonseca, M., The contribution of nuclear compartmentalization to gene regulation. Cell, 2002. 108(4): p. 513-21.

33. Cook, P.R., $A$ model for all genomes: the role of transcription factories. J Mol Biol, 2010. 395(1): p. 1-10.

34. Dunn, T.A., et al., A novel role of myosin $\mathrm{VI}$ in human prostate cancer. Am J Pathol, 2006. 169(5): p. 1843-54.

35. Loikkanen, I., et al., Myosin VI is a modulator of androgen-dependent gene expression. Oncol Rep, 2009. 22(5): p. 991-5.

36. Maddugoda, M.P., et al., Myosin VI and vinculin cooperate during the morphogenesis of cadherin cell cell contacts in mammalian epithelial cells. J Cell Biol, 2007. 178(3): p. 529-40.

37. Wang, H., et al., Lentivirus-Mediated Knockdown of Myosin VI Inhibits Cell Proliferation of Breast Cancer Cell. Cancer Biother Radiopharm, 2015. 30(8): p. 330-5.

38. Arden, S.D., et al., Loss of cargo binding in the human myosin VI deafness mutant (R1166X) leads to increased actin filament binding. Biochem J, 2016. 473(19): p. 3307-19.

39. Avraham, K.B., et al., The mouse Snell's waltzer deafness gene encodes an unconventional myosin required for structural integrity of inner ear hair cells. Nat Genet, 1995. 11(4): p. 369-75.

40. Sbalzarini, I.F. and P. Koumoutsakos, Feature point tracking and trajectory analysis for video imaging in cell biology. J Struct Biol, 2005. 151(2): p. 182-95.

41. Aaron, J., et al., Practical Considerations in Particle and Object Tracking and Analysis. Curr Protoc Cell Biol, 2019. 83(1): p. e88.

42. Batters, C., et al., Cloning, expression, and characterization of a novel molecular motor, Leishmania myosin-XXI. J Biol Chem, 2012. 287(33): p. 27556-66.

43. Toseland, C.P., Fluorescence to study the ATPase mechanism of motor proteins. EXS, 2014. 105: $p$. 67-86.

44. Toseland, C.P. and M.A. Geeves, Rapid reaction kinetic techniques. EXS, 2014. 105: p. 49-65.

45. Postma, M. and J. Goedhart, PlotsOfData-A web app for visualizing data together with their summaries. PLoS Biol, 2019. 17(3): p. e3000202. 